\title{
Half dose sugammadex combined with neostigmine is non-inferior to full dose sugammadex for reversal of rocuronium- induced deep neuromuscular blockade: a cost-saving strategy
}

\author{
Marie T. Aouad, Waseem S. Alfahel, Roland N. Kaddoum and Sahar M. Siddik-Sayyid
}

\begin{abstract}
Background: Sugammadex reverses the effect of rocuronium more rapidly and effectively than neostigmine, at all levels of neuromuscular blockade (NMB). However, its cost is prohibitive. The combination of half dose sugammadex with neostigmine would be non-inferior to full dose sugammadex for the reversal of deep NMB. This approach would reduce the cost of sugammadex while preserving its efficacy.

Methods: Patients were randomly allocated to receive sugammadex $4 \mathrm{mg} / \mathrm{kg}$ (Group S) or sugammadex $2 \mathrm{mg} / \mathrm{kg}$ with neostigmine $50 \mu \mathrm{g} / \mathrm{kg}$ and glycopyrrolate $10 \mu \mathrm{g} / \mathrm{kg}$ (Group NS) for reversal of rocuronium deep NMB. The primary outcome was the percentage of patients who recovered to 90\% Train of Four (TOF) ratio within 5 min. The non-inferiority margin was set at $10 \%$.

Results: Twenty eight patients were enrolled in each group. The number of patients who reached 90\% TOF ratio within 5 min was 27 out of 28 (96\%) in group S versus 25 out of 28 (89\%) in group NS by intention-to-treat (difference: $7 \%, 95 \% \mathrm{Cl}$ of the difference: $-9 \%$ to $24 \%$ ). The number of patients who reached $90 \%$ TOF ratio within 5 min was 26 out of 26 (100\%) in group S versus 23 out of 25 (92\%) in group NS by per-protocol (difference: 8\%, 95\% Cl of the difference: $-6 \%$ to $25 \%$ ).

Conclusions: Sugammadex $2 \mathrm{mg} / \mathrm{kg}$ with neostigmine $50 \mathrm{\mu g} / \mathrm{kg}$ was at worst $9 \%$ and $6 \%$ less effective than sugammadex $4 \mathrm{mg} / \mathrm{kg}$ by intention-to-treat and by per-protocol analysis respectively. Hence, the combination is non-inferior to the recommended dose of sugammadex.
\end{abstract}

Trial registration: Clinicaltrials.gov NCT 02375217, registered on February 11, 2015

Keywords: Neuromuscular blockade, Sugammadex, Rocuronium

\section{Background}

Sugammadex is a new reversal agent that works differently than cholinesterase inhibitors. It binds with high affinity to rocuronium or vecuronium in the blood, decreasing its plasma concentration and creating a gradient between the plasma and the neuromuscular junction. This process results in removing the non-depolarizing agent form the receptors as it follows the concentration gradient towards

\footnotetext{
* Correspondence: ss01@aub.edu.lb

Department of Anaesthesiology, American University of Beirut Medical Center, P.O. Box 11-0236, Beirut, Lebanon
}

the blood [1]. This novel reversal agent has changed established practices and resolved many clinical dilemmas, but has also created new challenges. It has been shown that within $5 \mathrm{~min}, 98 \%$ of patients would recover a train of four (TOF) ratio of 0.9 from a moderate rocuronium blockade (TOF of 2) with sugammadex versus only $11 \%$ of patients with neostigmine [2]. Sugammadex, if given in appropriate doses, has the ability to reverse the effect of rocuronium more rapidly and effectively than neostigmine, especially from deeper levels of neuromuscular blockade (NMB) [3]. Therefore, the availability of sugammadex has created a 
recent trend in the literature that advocates for the maintenance of deep levels of muscle relaxation till the end of surgery, mainly in laparoscopic surgery [4], yet the evidence on its beneficial effects is limited [5]. The claimed advantages of this practice are decreased postoperative shoulder pain [6] and improved surgical conditions [4]. Other advantages of sugammadex are the ability to reverse deep NMBs in unanticipated short surgeries without disrupting busy operating room schedules.

The superiority of sugammadex has even been established for shallower NMBs [7, 8]. A dose of $0.22 \mathrm{mg} / \mathrm{kg}$ suggamadex and $34 \mu \mathrm{g} / \mathrm{kg}$ neostigmine accelerates recovery from a TOF ratio of 0.5 to a TOF ratio of at least 0.9 in an average of $2 \mathrm{~min}$ [9]. However, the high cost of this drug is prohibitive and is a significant limitation for its routine use in many institutions, especially when relatively high doses are required. For the reversal of deep NMBs, defined as TOF $=0$ [10], the use of $4 \mathrm{mg} / \mathrm{kg}$ is recommended. We hypothesize that the combination of neostigmine with half dose sugammadex $(2 \mathrm{mg} / \mathrm{kg})$ would be non-inferior to the $4 \mathrm{mg} / \mathrm{kg}$ recommended dose in reversing rocuronium-induced deep NMB. Given the high cost of sugammadex, this multimodal approach would be an effective cost saving strategy, while preserving the advantages of this novel agent. Our primary outcome was the number of patients who reverse from a TOF count of 0 to a TOF ratio of 0.9 within $5 \mathrm{~min}$. Time of reversal to TOF ratio of 0.9 , patients with residual neuromuscular blockade defined as TOF less than 0.9 within $10 \mathrm{~min}$, time to extubation, TOF ratio at $5 \mathrm{~min}$, blood pressure and heart rate after reversal administration and number of sugammadex vials used were considered as secondary outcomes.

\section{Methods}

After obtaining the approval of the Institutional Review Board and written informed consent from patients, we conducted our study at the American University of Beirut Medical Center, Lebanon. The study was registered with the www.clinicaltrials.gov protocol registration system, principal investigator MTA, on February 11, 2015 (NCT 02375217). CONSORT statement for reporting noninferiority and equivalence trials were followed. Inclusion criteria were patients aged 18 to 70 years, of ASA classification I-III, undergoing elective surgery and requiring the use of muscle relaxants throughout the surgery. Exclusion criteria were the presence of renal dysfunction defined as creatinine $>1.2 \mathrm{mg} / \mathrm{dL}$ or known hepatic disease, obstructive sleep apnea, allergy to rocuronium or sugammadex, pregnant or obese patients (BMI $>35 \mathrm{~kg} / \mathrm{m}^{2}$ ), patients receiving medications known to interfere with the neuromuscular transmission, or having neuromuscular diseases.

Premedication with diazepam $5 \mathrm{mg}$ PO was given, standard monitors were applied and anesthesia was induced using midazolam 1-2 mg, lidocaine $1.5 \mathrm{mg} / \mathrm{kg}$, propofol $2 \mathrm{mg} / \mathrm{kg}$, fentanyl $100 \mu \mathrm{g}$ and rocuronium $0.6 \mathrm{mg} / \mathrm{kg}$ to facilitate tracheal intubation. Maintenance was achieved using sevoflurane $2 \%$, incremental doses of fentanyl and dexmedetomidine $0.5 \mathrm{mcg} / \mathrm{kg} / \mathrm{h}$. Dexmedetomidine was maintained till $90 \%$ recovery of TOF to avoid hand movements.

The depth of the NMB was monitored by means of kinemyography using a nerve stimulator attached to the proximal medial aspect of the forearm along the run of the ulnar nerve (GE Datex Ohmeda, M-NMT Module, Healthcare Finland Oy Helsinki, Finland). After the administration of propofol and before the administration of rocuronium, automatic calibration of the device was done and the supramaximal current of stimulation was set. TOF stimuli at the supramaximal current were applied at $20 \mathrm{~s}$ interval. The hand was lying freely on the arm board and kept warm by using a forced-air warming blanket. Rocuronium was given by incremental boluses of $10 \mathrm{mg}$ to maintain deep $\mathrm{NMB}$ $($ TOF count $=0)[10]$ till the end of surgery.

A research assistant randomly assigned patients to one of two groups according to computer generated table of random numbers. The group assignment was kept in opaque sealed envelopes which were opened sequentially before study drug administration. Study drugs were drawn in 2 separate syringes, were diluted to a total volume of $10 \mathrm{ml}$ with normal saline and were labeled with the randomization number. The anesthesiologist collecting the data and administering the reversal was blinded to group allocation. At the end of surgery, patients in group NS received sugammadex $2 \mathrm{mg} /$ $\mathrm{kg}$ and neostigmine $50 \mu \mathrm{g} / \mathrm{kg}$ with glycopyrrolate $10 \mu \mathrm{g} / \mathrm{kg}$ and patients in group $S$ received sugammadex $4 \mathrm{mg} / \mathrm{kg}$ and $10 \mathrm{~mL}$ of normal saline. The times from reversal administration to $90 \%$ recovery of TOF ratio, and to extubation were recorded. The number of patients who recovered $90 \%$ TOF ratio within 5 min was computed. Systolic blood pressure (SBP) and heart rate (HR) were recorded before, and after 1 and $5 \mathrm{~min}$ of reversal administration. The number of vials of sugammadex used was recorded. Patients who did not achieve $90 \%$ TOF ratio recovery within $10 \mathrm{~min}$ from study drug administration were identified as patients with residual blockade. For those patients, it was planned to proceed with unblinding and to supplement with additional sugammadex $2 \mathrm{mg} / \mathrm{kg}$ for patients in group NS. In the post anesthesia care unit (PACU) the reoccurrence of signs of residual neuromuscular blockade (rNMB) including nystagmus, laryngospasm, weakness, inability to sustain head lift, uncoordinated movements, or desaturation $\left(\mathrm{SpO}_{2}<94 \%\right)$ was monitored by one of the investigators who was blinded to group allocation. All patients were observed for at least one hour following which they were discharged if they met standard discharge criteria.

\section{Statistical Analysis}

Our primary outcome was the proportion of patients who achieved $90 \%$ recovery of TOF ratio within $5 \mathrm{~min}$ of study drug administration. Patients were included in 
the per-protocol analysis if the primary outcome measure was available and if the TOF count was truly 0 at the time of study drug administration. In the intention-to-treat analysis, all patients were included even if the TOF count was more than 0 . The choice of the non-inferiority margin was done according to the division of Anti-Infective Drug Products of the FDA published recommendations in 1992 for the selection of deltas for non-inferiority trials; one approach to setting the lower bound of the 95\% CI (the delta) would be to base this limit on the success rate achieved in the trial. The recommendations were for use of $10 \%, 15 \%$, and $20 \%$ deltas in trials with success rates of $\geq 90 \%, 80 \%-89 \%$ and $\leq 79 \%$, respectively [11]. The primary outcome was expressed as numbers and percentages. Confidence intervals (CI) for difference of percentages were calculated using Wilson's procedure without continuity correction (http://vassarstats.net/prop2_ind.html) [12]. The criterion for non-inferiority was considered to have been met if the lower limit of a 2-sided 95\% CI for the difference between groups was less than $10 \%$. For secondary outcomes, normally distributed data were reported as means \pm SD and confidence intervals of the differences and analyzed using Student $t$ test with equal variances. $P<0.05$ was considered significant. The data were tested for normality using the Kolmogorov-Smirnov normality test with Lilliefors correction and were considered normally distributed if $P>$ 0.05. All analyses were performed using SPSS (version 22, Chicago, IL).

Power analysis was based on non-inferiority. The results from a previous study showed that $98 \%$ of patients recover to TOF of 0.9 within 5 min after reversal of moderate rocuronium-induced NMB with sugammadex [2]. Assuming that $88 \%$ of patients would recover to TOF of 0.9 in the NS group, and a non-inferiority margin set at $10 \%$, a sample size of 25 in each group was calculated, with $\alpha=$ 0.025 (one sided) and $\beta=0.2$. To account for $10 \%$ dropout rate, we enrolled 56 patients.

\section{Results}

Fifty six patients fulfilled the inclusion criteria and signed the informed consent during the study period (February 2015-November 2015); 28 patients were enrolled in each group. No patients were excluded. All patients had a TOF of 0 before reversal administration except for three patients in group NS and 2 patients in group $\mathrm{S}$ who had one twitch. This twitch reappeared at the time of reversal administration. Therefore, the 5 patients were given the reversal as for deep blockade and were considered as protocol violations. They were included in the intention-to-treat analysis but not in the per-protocol analysis (Fig. 1).

Operations were a mix of orthopedic, laparoscopic, gynecological, urological, head and neck, and general surgical procedure. The characteristics of patients, anesthesia and surgery were comparable between the 2 groups (Table 1). An intention-to-treat analysis showed that the number of patients who reached TOF of 0.9 within $5 \mathrm{~min}$ was 27 out of $28(96 \%)$ in group S versus 25 out of $28(89 \%)$ in group NS (difference: $7 \%, 95 \% \mathrm{CI}$ of the difference: $-9 \%$ to $24 \%$ ). A perprotocol analysis by excluding patients with TOF of 1 showed that the number of patients who reached $90 \%$ TOF ratio within 5 min was 26 out of $26(100 \%)$ in group S versus 23 out of 25 (92\%) in group NS (difference: 8\%, 95\% CI of the difference: $-6 \%$ to $25 \%$ ) (Fig. 2). The time to achieve $90 \%$ TOF ratio was $180.9 \pm 96.8 \mathrm{~s}$ in group $\mathrm{S}$ and $228.2 \pm 83.9 \mathrm{~s}$ in group NS ( $P=0.06$, difference: $47.3 \mathrm{~s}, 95 \% \mathrm{CI}$ of the difference: -95.9 to $1.2 \mathrm{~s})$. The time from reversal to extubation was $504 \pm 186 \mathrm{~s}$ in group $\mathrm{S}$ and $544 \pm 176 \mathrm{~s}$ in group NS $(P$ $=0.5$, difference: $29.6 \mathrm{~s}, 95 \% \mathrm{CI}$ of the difference: -126 to 66.8 s) (Table 2). All patients recovered 90\% TOF ratio within $10 \mathrm{~min}$ and no rescue sugammadex was needed.

SBP values were comparable between the 2 groups at all time points. HR values were comparable before and after 5 min of reversal administration. However, HR after $1 \mathrm{~min}$ of reversal was higher in group NS than group S (Table 3). The number of sugammadex vials per patient was $2 \pm 0.5$ in group $S$ and 1.2. \pm 0.4 in group NS, $P<0.001$, difference: 0.82 vials, $95 \% \mathrm{CI}$ of the difference: 0.59 to 1.1 vials). All patients were discharged from PACU after one hour. No signs of recurarization or adverse events were recorded.

\section{Discussion}

We showed that sugammadex $2 \mathrm{mg} / \mathrm{kg}$ with neostigmine $50 \mu \mathrm{g} / \mathrm{kg}$ is non-inferior to sugammadex $4 \mathrm{mg} / \mathrm{kg}$ in reversing deep $\mathrm{NMB}$, as per-protocol and intention-to-treat analyses, with a similar proportion of patients recovering $90 \%$ TOF ratio within $5 \mathrm{~min}$ in both groups. The times to $90 \%$ recovery of TOF ratio and to extubation were comparable between the 2 groups. No cases of recurarization were detected in the PACU. A moderate and transient increase in HR was detected in the combination group.

Sugammadex reverses rocuronium blockade in a dosedependent manner [13] and achieves full recovery more rapidly than neostigmine [3]. Applying half the recommended dose to reverse deep NMB ( $2 \mathrm{mg} / \mathrm{kg}$ in group NS) brought the patient into a shallower level of blockade and the reversal action was efficiently completed with neostigmine. The speed of action of sugammadex offers a major advantage over neostigmine reversal which might take up to $15 \mathrm{~min}$ to achieve its peak effect [14]. This advantage was not lost in the combination group, since approximately $90 \%$ of patients achieved full recovery within only $5 \mathrm{~min}$, and $100 \%$ achieved full recovery in less than $10 \mathrm{~min}$ with no signs of rNMB. Despite the fact that the full effect of neostigmine may take more than ten minutes, its effect could start earlier. The onset of action of action of neostigmine starts after 2 min [14]. Moreover, in a previous study Schaller et al. [9] reported that $0.22 \mathrm{mg} / \mathrm{kg}$ suggamadex and $34 \mu \mathrm{g} / \mathrm{kg}$ neostigmine accelerates recovery from a TOF 


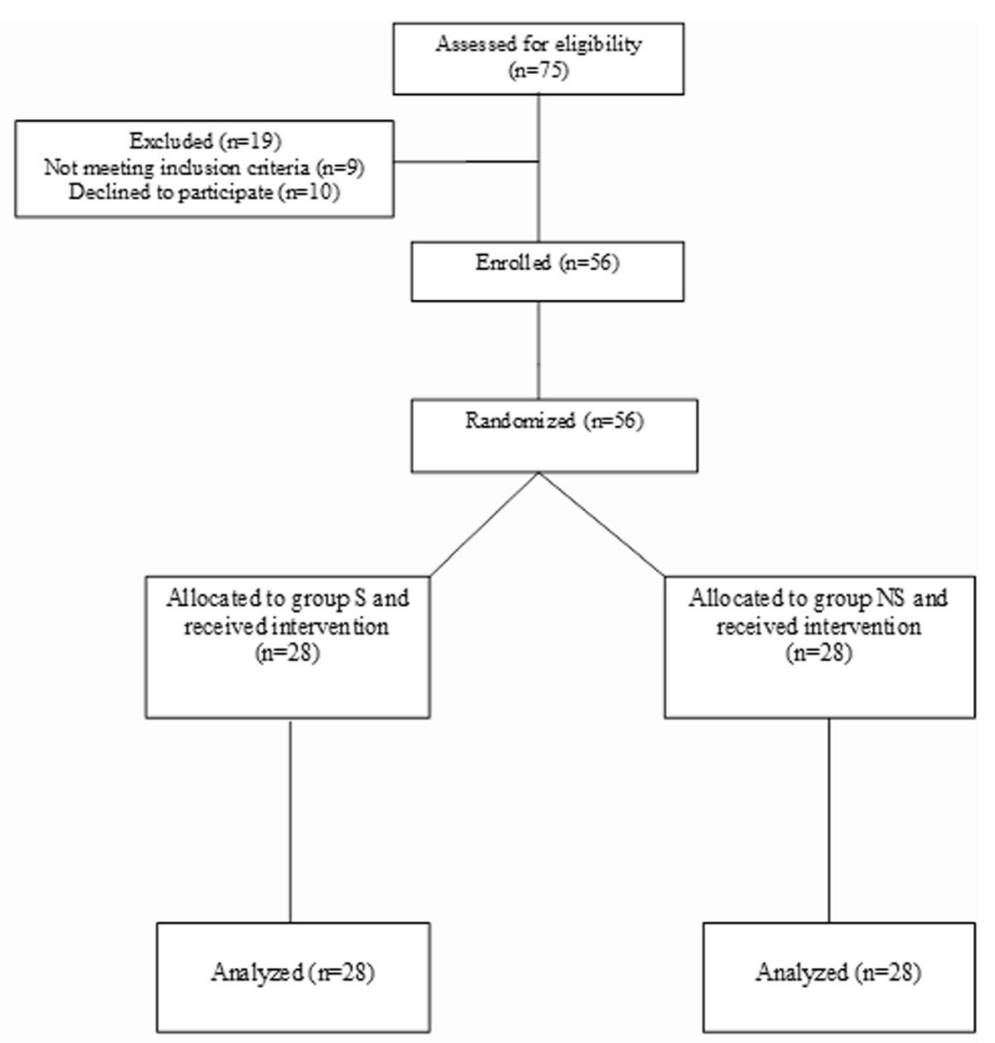

$S=$ Sugammadex, NS =Neostigmine and Sugammadex

Fig. 1 Flow chart

Table 1 Characteristics of patients and surgery

\begin{tabular}{lll}
\hline & Group S $(\mathrm{n}=28)$ & Group NS $(\mathrm{n}=28)$ \\
\hline Age (years) & $42.4 \pm 14.3$ & $40.6 \pm 13.5$ \\
Gender (M/F) & $15 / 13$ & $16 / 12$ \\
Weight (Kg) & $76.7 \pm 18.8$ & $72.9 \pm 11.2$ \\
Height (cm) & $168.3 \pm 13.6$ & $166.3 \pm 12.1$ \\
BMI (Kg/m $\left.{ }^{2}\right)$ & $26.9 \pm 5.7$ & $26.9 \pm 6.2$ \\
ASA Status & 14 & 13 \\
1 & 11 & 13 \\
2 & 3 & 2 \\
3 & & $100.0 \pm 48.9$ \\
Surgery time (min) & $109.1 \pm 84.2$ & $133.0 \pm 52.7$ \\
Anesthesia time $(\mathrm{min})$ & $142.4 \pm 91.8$ & $93.8 \pm 21.1$ \\
Total rocurunium $(\mathrm{mg})$ & $104.6 \pm 61.5$ & $65.8 \pm 35.9$ \\
Total rocuronium/h (mg/h) & $76.6 \pm 45.5$ & $1.3 \pm 0.4$ \\
Total rocuronium/Kg (mg/Kg) & $1.4 \pm 0.8$ & $39.9 \pm 10.9$ \\
Smax (mA) & $38.0 \pm 14.6$ & $35.8 \pm 0.6$ \\
Temperature at end of surgery $\left({ }^{\circ} \mathrm{C}\right)$ & $36.1 \pm 0.5$ & \\
\hline
\end{tabular}

Data are means \pm SD or numbers

$S$ Sugammadex, NS Neostigmine and Sugammadex, M Male, F Female, Smax Supramaximal current

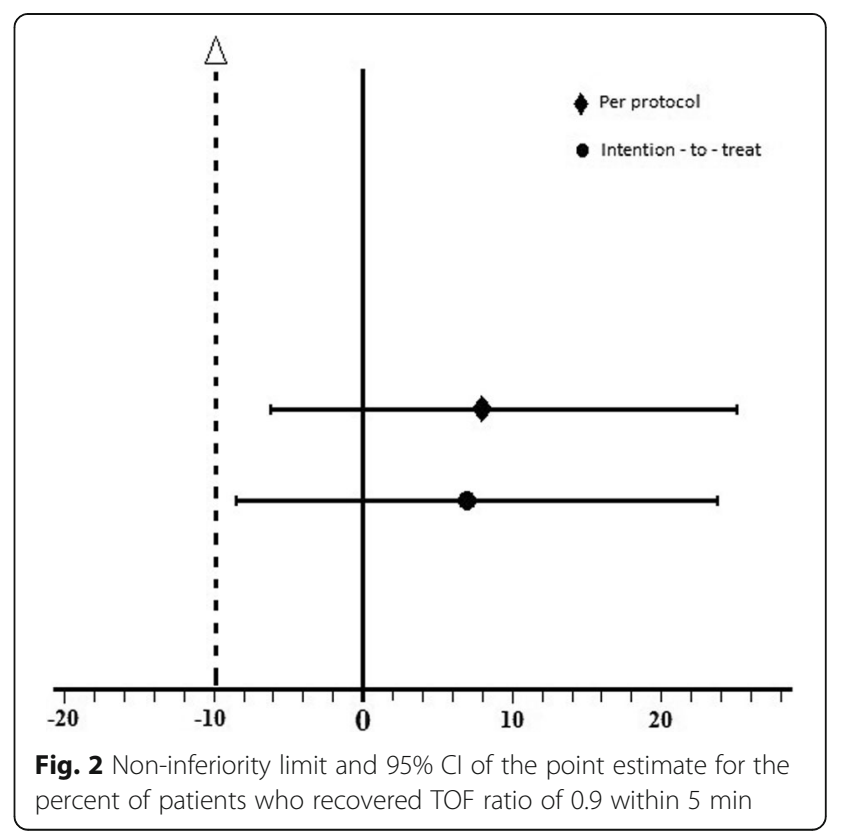


Table 2 Characteristics of neuromuscular block recovery

\begin{tabular}{|c|c|c|c|c|}
\hline & $\begin{array}{l}\text { Group S } \\
(n=28)\end{array}$ & $\begin{array}{l}\text { Group NS } \\
(\mathrm{n}=28)\end{array}$ & Difference & $\begin{array}{l}95 \% \text { confidence interval } \\
\text { of the difference }\end{array}$ \\
\hline $\begin{array}{l}\text { Number of patients with TOF } 0.9 \text { within } 5 \text { min } \\
\text { (intention-to-treat analysis) }\end{array}$ & $27(96)$ & $25(89)$ & $7 \%$ & $-9 \%$ to $24 \%$ \\
\hline $\begin{array}{l}\text { Number of patients with TOF } 0.9 \text { within } 5 \text { min } \\
\text { (per-protocol analysis) }\end{array}$ & $26(100)$ & $23(92)$ & $8 \%$ & $-6 \%$ to $25 \%$ \\
\hline Time to TOF 0.9 (sec) & $180.9 \pm 96.8$ & $228.2 \pm 83.9$ & -47.32 & -95.9 to 1.2 \\
\hline TOF ratio at $5 \mathrm{~min}$ & $0.96 \pm 0.11$ & $0.94 \pm 0.07$ & 0.02 & -0.03 to 0.07 \\
\hline Time to extubation (sec) & $502.5 \pm 192.0$ & $532.1 \pm 167.1$ & -29.6 & -126.0 to 66.8 \\
\hline Vials of sugammadex used per patient & $2 \pm 0.5$ & $1.2 \pm 0.4$ & 0.82 & 0.59 to 1.1 \\
\hline
\end{tabular}

The primary outcome was tested for non-inferiority and expressed as numbers (\%) and confidence intervals for the difference of percentages. The non-inferiority margin delta was set at $10 \%$. Non-inferiority was met since the lower limit of a 2 -sided $95 \%$ confidence interval is less than $10 \%$. Secondary outcomes were expressed as means \pm SD and analyzed using Student $t$ test

$S$ Sugammadex, NS Neostigmine and Sugammadex, TOF Train of Four

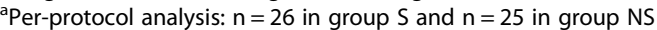

ratio of 0.5 to a TOF ratio of at least 0.9 in an average of 2 min but within 5 min for $95 \%$ of all treated patients.

Eikermann et al. [15] showed that the administration of neostigmine in the absence of residual NMB may impair genioglossus and diaphragm muscle function. Furthermore, Cammu et al. [16] showed that the administration of neostigmine following $2 \mathrm{mg} / \mathrm{kg}$ of sugammadex to reverse moderate neuromuscular blockade resulted in a weaker diaphragmatic electromyographic activity than sugammadex alone. However, in our study no rNMB blockade was detected neither clinically nor per the TOF. This might be explained by the fact that we administered only half the recommended dose of sugammadex which is not expected to produce complete recovery from deep NMB.

Sugammadex was used as a rescue drug in a patient who didn't return to full recovery after neostigmine [17]. Hence, the idea of sugammadex use for partial paralysis following insufficient recovery by neostigmine was raised. Subsequently, sugammadex was used as a rescue drug for incomplete reversal of rocuronium-induced NMB after neostigmine in a renal failure patient [18]. In a recent randomized trial, Cheong and colleagues investigated the combination of low dose sugammadex and neostigmine for reversal of rocuronium-induced moderate NMB [19]. The authors compared the time to $90 \%$ TOF ratio recovery in 4 reversal groups: sugammadex $2 \mathrm{mg} / \mathrm{kg}$ (S2), sugammadex $1 \mathrm{mg} / \mathrm{kg}$ (S1), sugammadex $1 \mathrm{mg} / \mathrm{kg}$ + neostigmine $50 \mu \mathrm{g} / \mathrm{kg}$ $(\mathrm{SN})$ and neostigmine $50 \mu \mathrm{g} / \mathrm{kg}$ alone $(\mathrm{N})$. At the time of reversal, TOF count was 1 or 2 . The total amount of rocuronium received per patient was not reported. Group SN showed significantly shorter recovery time than group S1 and N. Similar to our study, no statistically significant differences between the S2 and SN groups were observed. Furthermore, the recovery time from moderate NMB of $183 \mathrm{~s}$ in group S2 and 204 s in group SN were similar to the recovery times from deep NMB in our study (181 s. in group $\mathrm{S}$ and $228 \mathrm{~s}$. in group NS). Our study is the first to assess recovery from deep NMB with half dose sugammadex and neostigmine. Only sugammadex ampoules of $200 \mathrm{mg}$ are available. Therefore, the combination is not a useful cost containment strategy for the reversal of moderate NMB as in Cheong and colleagues, since using $1 \mathrm{mg} / \mathrm{kg}$ or $2 \mathrm{mg} / \mathrm{kg}$ would both result in opening one ampoule in an average $70 \mathrm{~kg}$ patient. Conversely, in our study, we demonstrated a substantial cost saving with the multimodal reversal from deep NMB. Using $2 \mathrm{mg} / \mathrm{kg}$ sugammadex instead of $4 \mathrm{mg} / \mathrm{kg}$ resulted in an approximate reduction of $50 \%$ in the number of vials used, i.e. using 1 vial instead of 2 vials for patients who weigh 51-100 kg. Other than the depth of the NMB, our methodology was different from the above mentioned

Table 3 Hemodynamic changes after administration of reversal agent

\begin{tabular}{|c|c|c|c|c|c|}
\hline & Group S $(n=28)$ & Group NS ( $n=28)$ & $P$ & Difference & $\begin{array}{l}95 \% \text { confidence interval } \\
\text { of the difference }\end{array}$ \\
\hline HR before reversal & $65.4 \pm 13.1$ & $68.4 \pm 12.2$ & 0.37 & -3.03 & -9.8 to 3.8 \\
\hline HR after $1 \mathrm{~min}$ & $64.7 \pm 15.3$ & $74.5 \pm 17$ & 0.03 & 9.82 & -18.5 to -1.1 \\
\hline HR after 5 min & $70.7 \pm 18.4$ & $70.5 \pm 17.6$ & 0.95 & 0.28 & -9.4 to 10 \\
\hline SBP before reversal & $118.6 \pm 20.5$ & $117.8 \pm 14.9$ & 0.88 & 0.75 & -8.8 to 10.3 \\
\hline SBP after 1 min & $121 \pm 17.1$ & $127.1 \pm 22.2$ & 0.26 & -6.03 & -16.7 to 4.6 \\
\hline SBP after 5 min & $123.9 \pm 20$ & $125.4 \pm 24.7$ & 0.8 & -1.5 & -13.4 to 10.4 \\
\hline
\end{tabular}

Data were expressed as means \pm SD and analyzed using Student $t$ test

S Sugammadex, NS Neostigmine and Sugammadex, HR Heart Rate, SBP Systolic Blood Pressure 
study. We used a non-inferiority design because it is more specific to our hypothesis. Also, we set the primary outcome as the percentage of patients who achieved full recovery within 5 min rather than the absolute recovery time value. We considered this primary outcome more meaningful because of the importance of shortening the residual paralysis period between the administration of the reversal and the return to $90 \%$ TOF ratio. Most of our patients in the combination group achieved full recovery within $5 \mathrm{~min}$ and preserved the characteristics of sugammadex recovery [2] despite the administration of approximately $0.8 \mathrm{mg} / \mathrm{kg} / \mathrm{h}$ of rocuronium and the maintenance of a deep level of NMB till the end of surgery.

The high cost of sugammadex has so far prevented its routine use. Ledowski and colleagues found that the cost of muscle relaxants and reversal increased from $42 \$$ to $127 \$$ per case after the introduction of unrestricted use of sugammadex [20]. Fuchs-Buder and colleagues assessed the feasibility of the routine use of sugammadex for reversal of rocuronium-induced NMB [21]. They concluded that despite the potential improvement in recovery times, its routine use could not be justified. In many institutions, restrictive strategies are adopted to decrease the use of expensive medications. A significant anesthesia cost-reduction and decreased utilization of cost-prohibitive agents was demonstrated after applying accessibility interventions [22]. More physicians have the tendency to use sugammadex when it is available and easily reachable. A retrospective study showed a significant increase of sugammadex use when its availability became unrestricted [23]. Should we find ways to cut down on the high cost of this drug, its use is expected to grow up significantly. In the present study, we introduced an approach that significantly decreased the cost of reversal without losing the benefits.

Sugammadex does not interact with the cholinergic system and consequently is not accompanied by the cardiovascular adverse effects that are seen with acetylcholinesterase inhibitors. In our study, the cardiovascular changes that followed neostigmine/glycopyrrolate administration were of limited amplitude and of small clinical significance in a relatively healthy population.

One limitation of our study is the inability to extrapolate our results to situations where sugammadex administration might be of a great help (bariatric surgeries). Of note, case of recurarization after administration of sugammadex for moderate NMB reversal in an obese patient was reported postoperatively [24]. Since sugammadex is not well studied in the obese population, we excluded patients with BMI more than $35 \mathrm{~kg} / \mathrm{m}^{2}$. Therefore, the generalizability of our results couldn't be extended to obese patients. Future studies in bariatric surgery might be needed. Another limitation is the relatively long time to extubation of approximately $500 \mathrm{~s}$ in both groups. This delay between $90 \%$ TOF ratio and extubation occurred because we kept an adequate depth of anesthesia by using dexmedetomidine during the recovery phase in order to secure motionless hands during TOF measurements. A third limitation is that the cost savings on sugammadex do not apply automatically and depend on the local situation in the single countries.

\section{Conclusion}

In conclusion, we demonstrated that the combination of neostigmine $50 \mu \mathrm{g} / \mathrm{kg}$ and sugammadex $2 \mathrm{mg} / \mathrm{kg}$ is at worse $9 \%$ and $6 \%$ less effective than sugammadex $4 \mathrm{mg} / \mathrm{kg}$ by intention-to-treat and per-protocol analysis respectively, in terms of the proportion of patients who achieved full recovery from rocuronium-induced deep NMB within $5 \mathrm{~min}$. Thus, the combination of neostigmine and half dose sugammadex is non-inferior to full dose sugammadex. Anesthesiologists might face the need to reverse deep NMB because of unanticipated short surgeries, inter-individual variability in the response to non-depolarizing muscle relaxants or as rescue for incomplete neostigmine reversal. Although cost price should not outweigh good clinical practice, our study has identified a safe cost saving strategy for the use of sugammadex for these clinical scenarios.

\section{Abbreviations}

NMB: Neuromuscular blockade; TOF: Train of Four; SBP: Systolic blood pressure; HR: Heart rate; PACU: Post anesthesia care unit; rNMB: Residual neuromuscular blockade

Acknowledgments

We thank Ms. Iman Jaafar for her help in data collection.

Funding

The study was funded by the American University of Beirut (Award Number: 102856).

Availability of data and materials

The data supporting our findings is available upon request from the corresponding author

Authors' contributions

MA helped design the study, conduct the study, analyze the data, and write the manuscript WA helped design the study, conduct the study, analyze the data, and write the manuscript RK helped design the study, conduct the study, analyze the data, and write the manuscript SS helped design the study, conduct the study, analyze the data, and write the manuscript. All authors read and approved the final manuscript.

\section{Competing interests}

The authors declare that they have no competing interests.

\section{Consent for publication}

Not applicable.

\section{Ethics approval and consent to participate}

This study was approved by the Institutional Review Board at the American University of Beirut (Reference number: ANES.MA.14).

Patients who fit the inclusion criteria of our study were approached by a research assistant who described the study in details, and explained its risks and benefits. The patients were assured of the confidentiality of the data and were told they could withdraw from the study at any time. The patients were also assured that not participating, or withdrawing after giving consent, would not affect the quality of care provided. The recruited patients all signed written informed consents. 


\section{Publisher's Note}

Springer Nature remains neutral with regard to jurisdictional claims in published maps and institutional affiliations.

Received: 23 November 2016 Accepted: 3 April 2017

Published online: 11 April 2017

\section{References}

1. Naguib M. Sugammadex: another milestone in clinical neuromuscular pharmacology. Anesth Analg. 2007;104:575-81.

2. Blobner M, Eriksson LI, Scholz J, Motsch J, Della Rocca G, Prins ME. Reversal of rocuronium-induced neuromuscular blockade with sugammadex compared with neostigmine during sevoflurane anaesthesia: results of a randomised, controlled trial. Eur J Anaesthesiol. 2010;27:874-81.

3. Della Rocca G, Pompei L, Pagan DEPC, Tesoro S, Mendola C, Boninsegni P, Tempia A, Manstretta S, Zamidei L, Gratarola A, P. Murabito P, Fuggiano L, Di Marco. Reversal of rocuronium induced neuromuscular block with sugammadex or neostigmine: a large observational study. Acta Anaesthesiol Scand. 2013; 57:1138-45.

4. Dubois PE, Putz L, Jamart J, Marotta ML, Gourdin M, Donnez O. Deep neuromuscular block improves surgical conditions during laparoscopic hysterectomy: a randomised controlled trial. Eur J Anaesthesiol. 2014;31:430-6.

5. Kopman AF, Naguib M. Laparoscopic surgery and muscle relaxants: is deep block helpful? Anesth Analg. 2015;120:51-8.

6. Lindekaer $\mathrm{AL}$, Halvor Springborg $\mathrm{H}$, Istre O. Deep neuromuscular blockade leads to a larger intraabdominal volume during laparoscopy. J Vis Exp. 2013; 76, e50045.

7. Pongracz A, Szatmari S, Nemes R, Fulesdi B, Tassonyi E. Reversal of neuromuscular blockade with sugammadex at the reappearance of four twitches to train-of-four stimulation. Anesthesiology. 2013;119:36-42.

8. Khuenl-Brady KS, Wattwil M, Vanacker BF, Lora-Tamayo Jl, Rietbergen H, Alvarez-Gomez JA. Sugammadex provides faster reversal of vecuroniuminduced neuromuscular blockade compared with neostigmine: a multicenter, randomized, controlled trial. Anesth Analg. 2010;110:64-73.

9. Schaller SJ, Fink H, Ulm K, Blobner M. Sugammadex and neostigmine dosefinding study for reversal of shallow residual neuromuscular block. Anesthesiology. 2010;113:1054-60.

10. Kopman AF, Brull SJ. Low-dose sugammadex reversal: there is no such thing as a free lunch. Anesthesiology. 2013;119:10-2.

11. Powers JH, Ross DB, Brittain E, Albrecht R, Goldberger MJ. The United States Food and Drug Administration and noninferiority margins in clinical trials of antimicrobial agents. Clin Infect Dis. 2002;34:879-81.

12. Newcombe RG. Interval estimation for the difference between independent proportions: comparison of eleven methods. Stat Med. 1998;17:873-90.

13. Puhringer FK, Gordon M, Demeyer I, Sparr HJ, Ingimarsson J, Klarin B, van Duijnhoven W, Heeringa M. Sugammadex rapidly reverses moderate rocuronium- or vecuronium-induced neuromuscular block during sevoflurane anaesthesia: a dose-response relationship. Br J Anaesth. 2010;105:610-9.

14. Calvey TN, Wareing M, Williams NE, Chan K. Pharmacokinetics and pharmacological effects of neostigmine in man. Br J Clin Pharmacol. 1979;7:149-55.

15. Eikermann M, Fassbender P, Malhotra A, Takahashi M, Kubo S, Jordan AS, Gautam S, White DP, Chamberlin NL. Unwarranted administration of acetylcholinesterase inhibitors can impair genioglossus and diaphragm muscle function. Anesthesiology. 2007;107:621-9.

16. Cammu G, Schepens T, De Neve N, Wildemeersch D, Foubert L, Jorens PG. Diaphragmatic and intercostal electromyographic activity during neostigmine, sugammadex and neostigmine-sugammadex-enhanced recovery after neuromuscular blockade: A randomised controlled volunteer study. Eur J Anaesthesiol. 2017:34:8-15.

17. De Menezes CC, Peceguini LA, Silva ED, Simoes CM. Use of sugammadex after neostigmine incomplete reversal of rocuronium-induced neuromuscular blockade. Rev Bras Anestesiol. 2012;62:543-7.

18. Lobaz S, Sammut M. Damodaran A. BMJ Case Rep: Sugammadex rescue following prolonged rocuronium neuromuscular blockade with 'recurarisation' in a patient with severe renal failure; 2013.

19. Cheong SH, Ki S, Lee J, Lee JH, Kim MH, Hur D, Cho K, Lim SH, Lee KM, Kim $Y J$, Lee $\mathrm{W}$. The combination of sugammadex and neostigmine can reduce the dosage of sugammadex during recovery from the moderate neuromuscular blockade. Korean J Anesthesiol. 2015;68:547-55.

20. Ledowski T, Hillyard S, Kozman A, Johnston F, Gillies E, Greenaway M, Kyle BC. Unrestricted access to sugammadex: impact on neuromuscular blocking agent choice, reversal practice and associated healthcare costs. Anaesth Intensive Care. 2012;40:340-3.

21. Fuchs-Buder T, Meistelman C, Schreiber JU. Is sugammadex economically viable for routine use. Curr Opin Anaesthesiol. 2012;25:217-20.

22. Tabing AK, Ehrenfeld JM, Wanderer JP. Limiting the accessibility of costprohibitive drugs reduces overall anesthetic drug costs: a retrospective before and after analysis. Can J Anaesth. 2015;62:1045-54.

23. Watts RW, London JA, van Wijk RM, Lui YL. The influence of unrestricted use of sugammadex on clinical anaesthetic practice in a tertiary teaching hospital. Anaesth Intensive Care. 2012;40:333-9.

24. Le Corre F, Nejmeddine S, Fatahine C, Tayar C, Marty J, Plaud B. Recurarization after sugammadex reversal in an obese patient. Can J Anaesth. 2011;58:944-7.

\section{Submit your next manuscript to BioMed Central and we will help you at every step:}

- We accept pre-submission inquiries

- Our selector tool helps you to find the most relevant journal

- We provide round the clock customer support

- Convenient online submission

- Thorough peer review

- Inclusion in PubMed and all major indexing services

- Maximum visibility for your research

Submit your manuscript at www.biomedcentral.com/submit
C) Biomed Central 